International Journal of Trend in Scientific Research and Development (IJTSRD)

Volume: 3 | Issue: 3 | Mar-Apr 2019 Available Online: www.ijtsrd.com e-ISSN: 2456 - 6470

\title{
A Crowd Sourcing to Track Individuals in Highly Active Industries
}

\author{
R. Mahalakshmi, S. Janani, R. Sivasankari \\ Department of Computer Science and Engineering, \\ G.K.M. College of Engineering and Technology, Chennai, Tamil Nadu, India
}

\begin{abstract}
How to cite this paper: $\mathrm{R}$. Mahalakshmi | S. Janani | R. Sivasankari "A Crowd Sourcing to Track Individuals in Highly Active Industries" Published in International Journal of Trend in Scientific Research and Development (ijtsrd), ISSN: 24566470, Volume-3 | Issue-3, April 2019, pp.336-338, URL: http://www.ijtsrd.co $\mathrm{m} /$ papers/ijtsrd 227 77.pdf

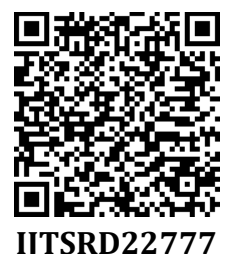

Copyright (C) 2019 by author(s) and International Journal of Trend in Scientific Research and Development Journal. This is an Open Access article distributed under the terms of the Creative Commons

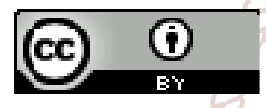
Attribution License (CC BY 4.0) (http://creativecommons.org/licenses/ by $/ 4.0$ )

\section{INTRODUCTION}

Positioning technologies have reached a significant level of maturity over the last years there is still no adequate solution for providing accurate positioning services across large and active industries. Tracking the workers in a construction site is much more challenging than indoor positioning mainly due to the many moving parts and the fast large-scale changes that occur in these complex environments. Currently, there is no system that allows for workers to be tracked reliably and robustly during all phases of construction. This paper presents a concept or idea to track employee in highly active industries. A positioning system to track the employee using occlusion maps. The main aim of the project is to replace the existing indoor positioning system (CCTV cameras). The proposed project is designed to provide a system that can monitor the location of workers to indicate working hazards (e.g., red and green zones), which can be tracked individually

\section{RELATED WORK}

The more recent RGB-W system from when vision meets wireless by A. Alahi, A. Haque, and L. Fei-Fe, 2015 also uses wireless signals emitted by people's mobile phones in combination with cameras to track and identify people. The authors show how the wireless signals can be used as a rough proxy for depth information which allows them to achieve better localization accuracy. S. Papaioannou, H. Wen, A. Markham, and N. Trigoni, 2014 Fusion of radio and camera sensor data for accurate indoor positioning, The Radio and Vision Enhanced Localization (RAVEL) system fuses anonymous visual detections captured by a stationary camera with Wi-Fi readings to track multiple people moving inside an area with CCTV coverage. D. Lymberopoulos, J. Liu, X. Yang, R. R. Choudhury, S. Sen, and V. Handzinski, "Microsoft indoor localization competition: Experiences and lessons learned, the most recent positioning systems that make use of radio, inertial- and visual- sensing (i.e., using a stationary camera) to track multiple people. R. Mandeljc, S. Kovacic, M. Kristan, and J. Pers, "Tracking by identification using computer vision and radio," Sensors was evaluated using only UWB radios which exhibit sub-meter accuracy and there is no indication of how this method will perform with radios of lower accuracy (i.e., Wi-Fi).2014 M. Shah, J. Deng, and B. Woodford, "Video background modelling: Recent approaches, issues and our proposed techniques," Effective and efficient background subtraction is important to a number of computer vision tasks. We introduce several new techniques to address key challenges for background modelling using a Gaussian mixture model (GMM) for moving objects detection in a video acquired by a static camera. proposed model is rigorously tested and compared with several previous models and has shown significant performance improvements.2012 J. Ning, L. Zhang, D. Zhang, and $\mathrm{C}$. $\mathrm{Wu}$, "Robust mean-shift tracking with corrected background-weighted histogram," The background-weighted histogram (BWH) algorithm proposed in attempts to reduce the interference of background in target localization mean shift tracking. The experimental results show that CBWH can lead to faster convergence and more accurate localization 
than the usual target representation in mean shift tracking. algorithm can still robustly track the object, which is hard to achieve by the conventional target representation.

\section{PROPOSED SYSTEM}

In this project, Google map is used to track the employee location. Once the employee signed in to the account, admin can access the employee location. Admin would be able to surveillance the employee anywhere in the world without spending more cost. Although admin can easily do micro monitoring as well, if the employee could not access for long time admin will get some kind of alert. If any employee tends to enter any restricted area, the admin can easily track the information of the employee with the help of registered employees photograph. It helps in less weight age of document. Alert notification is provided to admin and Geo fencing algorithm has been used.

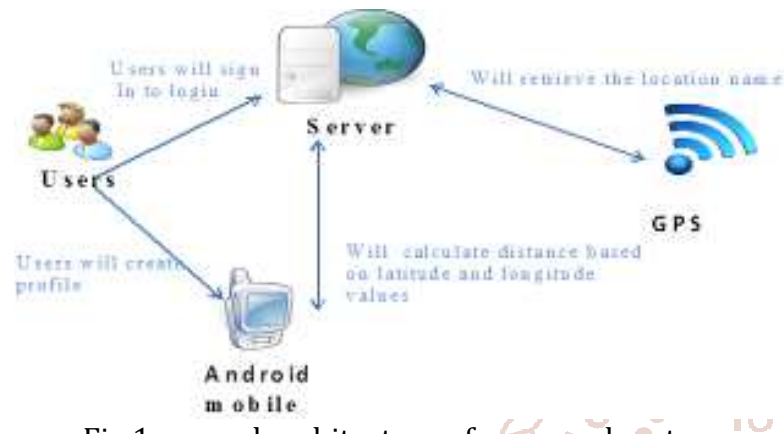

Fig.1 general architecture of proposed system Even if the target is not well initialized, the proposed

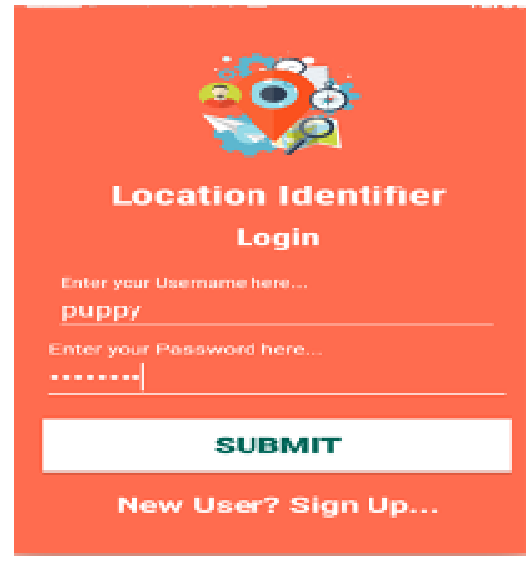

Fig.3 login page

\section{Admin module}

$>$ Employee Tracking:

In this Module, admin have login to process the employee tracking by approving the new user registration and check the status of the employee by tracking and control.

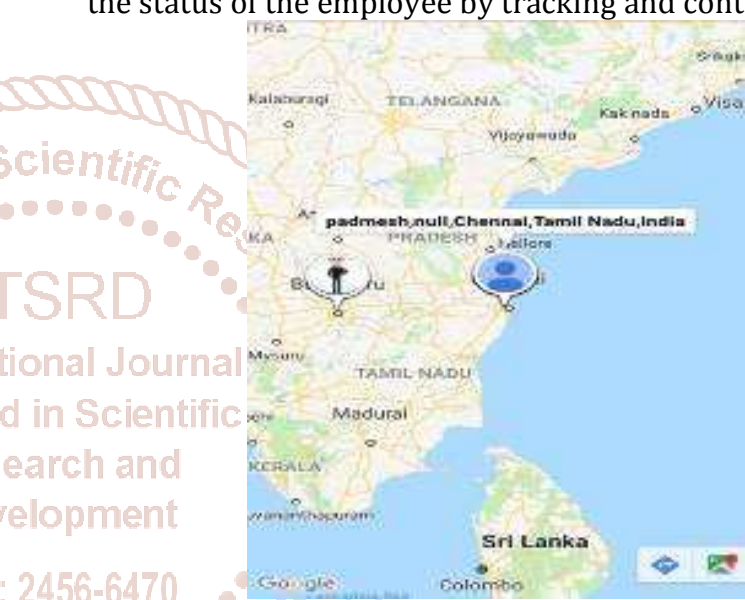

1. Creating new account

In this module, we create a new account for employee tracking on both user and admin bases. The user personal details ex: name, id, department, gender, phone number and etc. Admin details like id, password. User given details are used in the database.

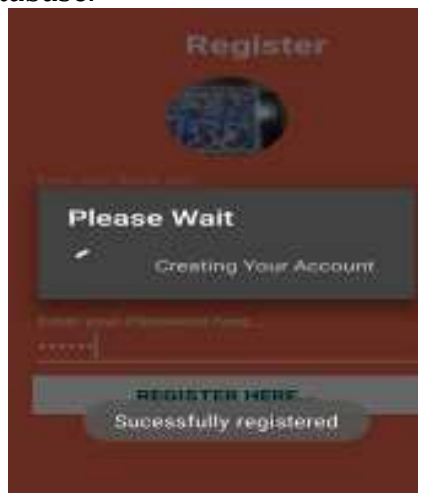

Fig.2 registration

\section{Login}

The module describes signup page containing email id or user name, password and confirm password. These are retrieved from the database and combine based on user input if it matches user name and password in the app otherwise alert messages will be displayed to the user. fig.4 tracking people

Attendance process:

In this module the employee attendances are automatically calculated with their tracking location inside the site area Attendance can be calculated automatically when they reach their area of field. So it will be easy to track all the employee.

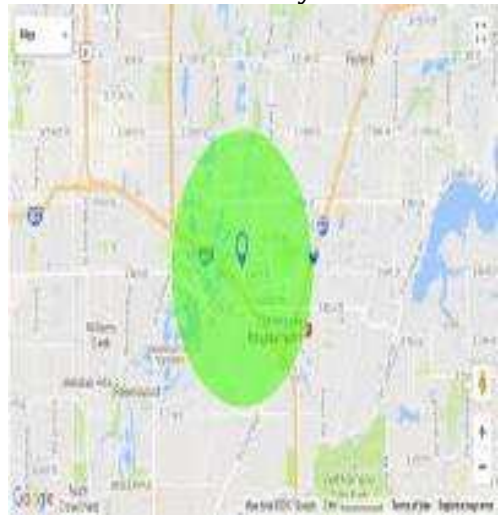

fig.5 attendance process

\section{3. user module}

$>$ Tracking process

In this module, the employee is tracked automatically with the help of their location in the site and there in and out time is also calculated automatically. There area of work and restricted areas are also detected in the map 
$>$ Notification process:

The module deals with the check in and checkout notification of the employee according to their current date and stores it in the database for the fetching process of the data.

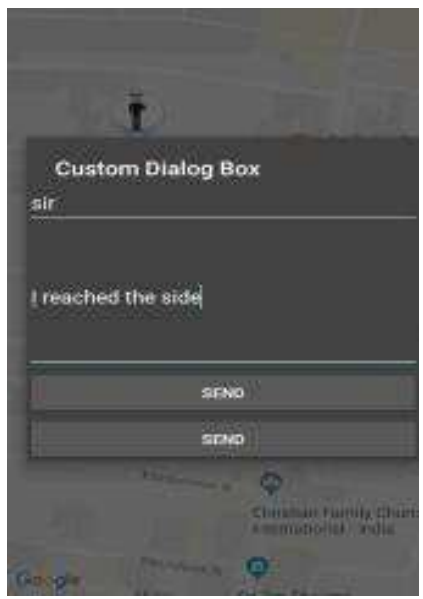

Fig.6 notification page

\section{HARDWARE PHASE}

\section{$>$ RA M}

3GB RAM minimum,8 GB RAM recommended, $1 \mathrm{~GB}$ for android emulator.

$>$ Hard disk

500 MB disk space for Android Studio, at least 1.5 GB for Android SDK, emulator system images, and caches

$>$ Mobile

Emulator (Above Kitkat Version).

Processor

Intel 64-bit processor is used to run the project.

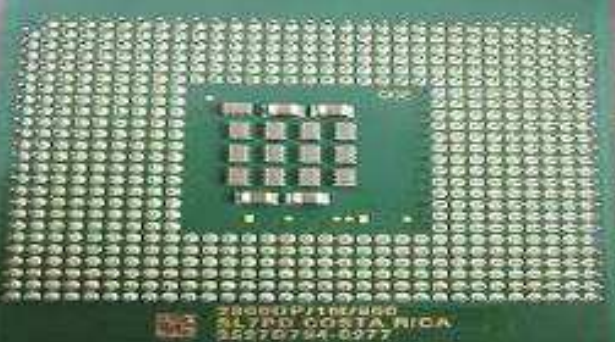

fig.7 intel-64bit processor

\section{SOFTWARE PHASE}

$>$ Java Development Kit (JDK) 8

The Java Development Kit (JDK) is an implementation of either one of the Java Platform, Standard Edition, Java Platform, Enterprise Edition, or Java Platform, i

MySQL

MySQL is written in C and C++. Its SQL parser is written in yacc, but it uses a home-brewed analyzer. it is free and open source.

\section{CONCLUSION}

In this paper we proposed a multi-modal positioning system for highly dynamic environments. We showed that it is possible to adapt particle filters-traditionally used to discern tracks using anonymous measurements-in order to both identify and tracking the people by using the GPS devices monitored by CCTV and holding mobile devices. We further showed that there is significant scope for automatically training the various sensor modalities, and this proved particularly useful in rapidly changing environments. Additionally, we showed that the use of social forces in dynamic industrial environments is highly beneficial and improves the tracking accuracy. Our experiments showed that even without training, our online approach achieves similar positioning accuracy to the existing off line RAVEL approach; with training the positioning error is decreased by a further 50 percent. We also showed that the proposed technique robust in scenarios with visual and inertial noise.

\section{REFERENCES}

[1] Blackman S. and R. Popoli, Design and Analysis of Modern Tracking Systems. Norwood, MA, USA: Artec House, 1999.

[2] A. Doucet, N. De Freitas, K. P. Murphy, and S. J. Russell, "Rao Black wellised particle filtering for dynamic Bayesian networks," in Proc. 16th Conf. Uncertainty Artif . Intell., 2000, pp. 176-183. [Online].

[3] A. Doucet, N. De Freitas, and N. Gordon, Sequential Monte Carlo Methods in Practice. Berlin, Germany: Springer-Verlag, 2001.

[4] S. Papaioannou, H. Wen, A. Markham, and N. Trigoni, "Fusion of radio and camera sensor data for accurate indoor positioning," in Proc. IEEE 11th Int. Conf. Mobile Ad Hoc Sensor Syst., Oct. 2014, pp. 109-117.

[5] A. Alahi, A. Haque, and L. Fei-Fei, "RGB-W: When vision meets wireless," in Proc. IEEE Int. Conf. Comput. Vis., Dec. 2015, pp. 3289-329. 